\title{
¿CÓMO PERCIBEN LOS ESTUDIANTES UNIVERSITARIOS LA ENSEÑANZA DE LA FILOSOFÍA, SEGÚN SUS EXPERIENCIAS EN LA EDUCACIÓN DIVERSIFICADA COSTARRICENSE? ${ }^{1}$
}

\author{
Álvaro Carvajal Villaplana* \\ Jacqueline García Fallas**
}

Resumen: En 1998 se inicia el desarrollo de la investigación "La situación de la Enseñanza de la Filosofía en la Educación Diversificada costarricense", en el Instituto de Investigaciones Filosóficas de la Universidad de Costa Rica. En este artículo se analizan los resultados obtenidos de una encuesta administrada a estudiantes que cursan los estudios generales o su equivalente en tres de las universidades públicas. Esta encuesta aporta información sobre la percepción estudiantil entorno a la enseñanza de la filosofía, la cual emana de las experiencias construidas en el quinto año de la educación diversificada y en el primero año de su ingreso a la universitaria, esta última es la experiencia más cercana que tiene la población estudiantil en relación con la enseñanza y el aprendizaje de la filosofía. Estos resultados aportan aspectos significativos para reflexionar sobre la pertinencia de la educación filosófica en Costa Rica.

Palabras Claves: CREENCIAS Y CONCEPCIONES/ EDUCACIÓN DIVERSIFICADA/ FORMACIÓN EN HUMANIDADES/ ENSEÑANZA DE LA FILOSOFÍA/

\begin{abstract}
In this article the results of an survey applied to students who attend the general studies or their equivalent in three of the public universities are analyzed. This survey contributes information on the student perception in relation to its experiences constructed in the diversified education and in the University's education, regarding the subject of philosophy. This information allows to value the necessity to reframe this subject's educative curriculum in the diversified education's cycle.
\end{abstract}

Keywords: BELIEFS AND CONCEPTS/ DIVERSIFIED EDUCATION/ TEACHING OF HUMANITIES/ PHILOSOPHY TEACHING / HIGHER EDUCATION/

\section{Antecedentes}

Desde 1968 el Ministerio de Educación Pública formalizó la enseñanza de la filosofía en los colegios académicos, por lo que dicha enseñanza comprende la mayor parte de las instituciones del sistema educativo nacional, en el ámbito urbano y rural. El crecimiento

\footnotetext{
*Licenciado en Filosofía de la Universidad de Costa Rica. Actualmente es candidato a Doctor en Filosofía de la Universidad Carlos III de Madrid. Profesor de Filosofía en la Escuela de Estudios Generales, en la Escuela de Filosofía de la Universidad de Costa Rica y en el Instituto Tecnológico de Costa Rica. acarvaja@cariari.ucr.ac.cr

**Doctora en Educación de la Universidad de Costa Rica. Profesora de Filosofía en la Escuela de Filosofía, de Teoría de la educación en la Escuela de Formación Docente de la Universidad de Costa Rica. igarcía@cariari.ucr.ac.cr
}

Artículo recibido: 8 de febrero, 2004

Aprobado: 28 de junio, 2004

\footnotetext{
${ }^{1}$ En el análisis de los datos de la encuesta participó el profesor Lic. Mario Alfaro; asimismo, en la recolección de los datos y discusiones de investigación se involucraron también los profesores Dr. Manuel Triana y Lic. Carlos Alberto Rodríguez.
} 
poblacional tuvo impacto en el aumento de centros educativos para el nivel de secundaria, lo cual exigió una notabilísima ampliación del número de profesionales en todas las disciplinas del currículo oficial. La filosofía es una asignatura que corrió con la misma suerte, pero no ha tenido la misma atención que las demás asignaturas, especialmente en lo que se refiere a la formación de docentes, la renovación del currículo y la producción de materiales didácticos acordes con las necesidades socioculturales emergentes. Lo anterior, se hace más grave en el marco de los colegios académicos ubicados en zonas rurales; aunque, esto no significa que estén cubiertos los de zonas semiurbanas y urbanas en su totalidad.

A propósito de la formación de docentes, las lecciones en los colegios han sido encargadas, en su mayoría, a docentes que carecen de formación básica en el campo de la filosofía. El currículo vigente autorizado por el Ministerio de Educación Pública requiere de una revisión pertinente sobre temas, contenidos y metodologías, que respondan a una revisión del proceso pedagógico y su vinculación con el sujeto de la educación diversificada, es decir con jóvenes que enfrentan cambios físicos, emocionales, psicológicos, así como la necesidad de involucrarse afectivamente con otras personas y son parte de contextos socioculturales específicos, aparecen exigencias laborales, entre otras características que deberían orientar la formación filosófica. Sin embargo, para el desarrollo de los procesos de enseñanza y aprendizaje se cuenta apenas con dos textos oficiales, los cuales no han sido revisados ni reelaborados en 24 años. A lo anterior se añade la falta de un asesor nacional destacado por el Ministerio para dar seguimiento al personal docente en los colegios que imparten filosofía, lo cual se dificulta, además, porque las direcciones regionales tampoco cuentan con asesores en esta asignatura.

Como fue demostrado por la investigación "La situación de la enseñanza de la filosofía en la educación diversificada costarricense" (1998-2000), en zonas distantes, las lecciones de filosofía son asumidas por docentes de otras áreas académicas, como estudios sociales, música, religión, psicología, entre otras. Incluso, se dan casos de nombramientos de personas recién egresadas de la educación secundaria, lo que agrava el problema de los procesos pedagógicos, de enseñanza y aprendizaje, porque se trata de un personal, en la mayoría de los casos, sin formación en filosofía ni en educación.

Este panorama dificulta el grado de dominio de conocimientos y estrategias pedagógicas que se pueden poner en práctica en las aulas de los niveles de la educación diversificada, así como la pertinencia de los contenidos curriculares.

El diagnóstico aludido en la investigación mencionada, traza los caminos a seguir en cuanto al mejoramiento de los procesos para la enseñanza y el aprendizaje de la filosofía en 
la educación diversificada. Los docentes son los principales gestores del mejoramiento de la calidad de dichos procesos, razón por la cual es importante atender las necesidades de formación y actualización de aquellos que están en servicio, sin tener dominio acreditado en conocimientos filosóficos. Estos docentes son personas nombradas en el Ministerio de Educación Pública, algunos con experiencia de muchos años. No obstante, es necesario partir de la visión de la población estudiantil que ha tenido la experiencia de participar en las lecciones de filosofía, recientemente, en la educación diversificada, y, relacionar esa experiencia con la que se tiene en las aulas universitarias, cuando realizan sus cursos humanísticos, o propiamente de Filosofía. Por esta razón, este artículo analiza la visión de los estudiantes y no la de los docentes.

El propósito de realizar una encuesta a estudiantes universitarios que cursan estudios de filosofía en tres universidades públicas de Costa Rica reside en contar con un criterio cualitativo al partir de las percepciones que emergen del proceso pedagógico en la asignatura de Filosofía, lo cual es un insumo para la reflexión sobre la situación de la enseñanza de la filosofía en la educación diversificada. Por otra parte, permite relacionar la enseñanza de la filosofía en la educación diversificada con la experiencia de sus primeros encuentros con la asignatura en la formación universitaria, y entre las tres universidades seleccionadas para el estudio. La información obtenida sirvió para elaborar un instrumento más acabado para ser aplicado a los estudiantes de secundaria, cuya información es objeto de atención en la investigación mencionada.

La sociedad costarricense requiere que los aspectos críticos, integradores y creativos que aporta la Filosofía se hagan presentes de manera adecuada en la formación recibida por los estudiantes de secundaria. Estos aspectos son afines a la filosofía como disciplina, y son requeridas en un sistema educativo en el que se ofrece la posibilidad de lograr una visión de conjunto a partir de la formación filosófica y su incidencia en los procesos socioculturales de la sociedad actual. En este sentido valga recuperar la visión del filósofo norteamericano John Dewey (1998), quien a principio del siglo XX abogaba por una formación humanista y pragmática que favoreciera la construcción de una sociedad democrática y libre.

\section{Las creencias y concepciones para transformar los escenarios educativos}

La investigación educativa en las últimas dos décadas del siglo $\mathrm{XX}$, ha fundamentado una perspectiva de análisis que se enfoca en el estudio de las creencias y concepciones de 
los actores educativos, entre ellos los estudiantes. Esta perspectiva reconoce que las creencias y las concepciones pueden entenderse como construcciones personales realizadas a partir de experiencias cotidianas que provienen de entornos sociales específicos, las cuales se producen mediante actividades o prácticas culturales.

Estas creencias o concepciones constituyen la forma de expresión de la individualidad de la persona que las construye, aunque, al mismo tiempo, reflejan el conocimiento compartido con el grupo social de su pertenencia. El individuo no construye su representación en solitario ni sobre la base de experiencias idiosincrásicas, sino que el hecho de formar parte de grupos y espacios sociales, introduce elementos supraindividuales en el estudio de sus representaciones.

La construcción de creencias y concepciones está fuertemente orientada por las actividades (prácticas culturales) que el individuo realiza en su grupo, ocurre en un contexto de relación y de comunicación interpersonal (formatos de interacción) que trasciende la dinámica interna de la construcción personal, es decir en el espacio de mediación pedagógica en el aula. Las actividades que se llevan a cabo en esos contextos suelen estar socioculturalmente definida por: el juego, el estudio, el trabajo, la profesión y la paternidad, entre otros.

La síntesis y apropiación personal de conocimiento que se expresan por medio de creencias y concepciones, se ven propiciadas por las pautas estructuradas (prácticas) y la red de interacciones interpersonales que constituyen las manifestaciones funcionales de la cultura. Es decir, éstas se construyen principalmente a partir de experiencias directas, compartidas o no con los otros, o bien a partir de las experiencias obtenidas durante la observación del comportamiento ajeno. Facilitan la percepción de relaciones entre sucesos y aumentan la capacidad comprensiva. Cumplen un papel fundamental en la constitución de la visión de mundo, con la cual se lee y se otorga sentido a la experiencia.

En relación con la información suministrada por la encuesta aplicada a estudiantes universitarios, en un momento de transición entre el proceso de enseñanza y aprendizaje de la educación diversificada y el que le ofrece su inserción en la educación superior, permite identificar cuáles creencias y concepciones surgen de las experiencias de formación que responden al campo de la filosofía, las cuales responden a aspectos generales propios del sistema educativo nacional, la formación docente y el currículo oficial, así como a otros más específicos, la relación de lo que se enseña y se aprende en filosofía con lo que la población estudiantil necesita y espera de los procesos pedagógicos en esta asignatura, es decir del encuentro de esta asignatura con el sujeto de la educación diversificada. 


\section{Percepciones y creencias de la población estudiantil universitaria sobre sus experiencias de la filosofía en el colegio y en la universidad.}

Como se indica anteriormente estas percepciones y creencias emanan de una encuesta aplicada a estudiantes universitarios que han concluido la educación diversificada e inician la educación superior, tiene como punto de interés sus experiencias acerca de la filosofía. La encuesta se realizó entre los meses de octubre y noviembre de 1999, en la Universidad de Costa Rica (UCR), el Instituto Tecnológico de Costa Rica (ITCR) y la Universidad Nacional (UNA). En la UCR se considera el Curso Integrado de Humanidades (Opciones Regular y Seminario Participativo), en el ITCR el curso Seminario de Estudios Filosóficos e Históricos, y en la UNA los Núcleos Temáticos con énfasis en filosofía.

\subsection{Características de la población estudiantil.}

El total de estudiantes entrevistados fue de 390, de estos 254 son de la UCR (en razón de tener el mayor número de estudiantes), en el ITCR 49, y en la UNA 107. Del total de entrevistados 189 son hombres y 191 mujeres, lo que en términos relativos representa el $50 \%$ para cada género, como se ilustra en el gráfico No. 1.

\section{Gráfico $N^{\circ} 1$}

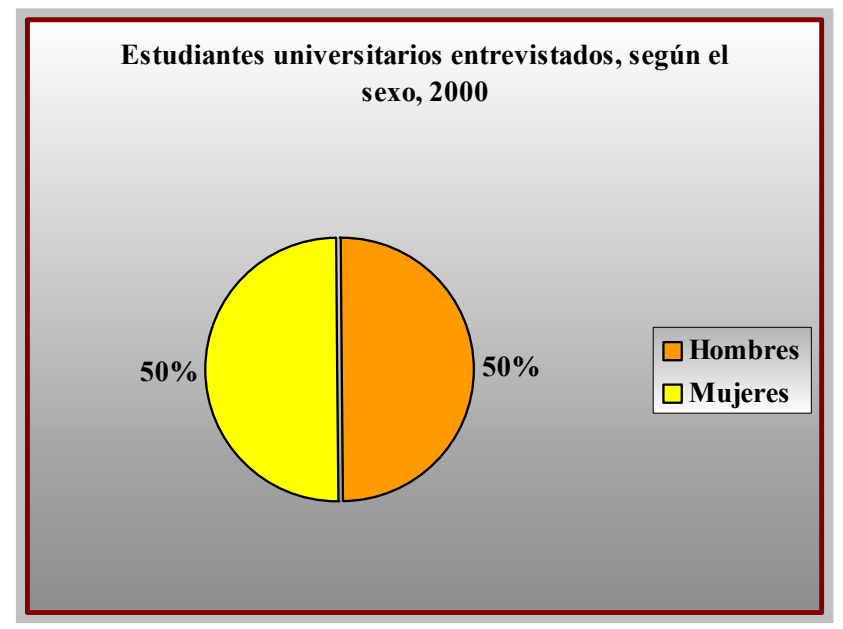

La mayoría de los estudiantes se encuentran en un rango de edad entre 17 y 20 años (317), 56 están en el segmento de 21 a 25 años y con más de 25 suman un total de 11 , y 6 no respondieron.

Un dato interesante que arroja la encuesta es que la mayoría, un $70 \%$, de los estudiantes entrevistados proceden de colegios públicos, de 271 estudiantes, 237 son de 
colegios académicos y 33 de colegios técnicos; tan solo el 17\% (es decir 69 estudiantes) provienen de colegios privados y 3 no responden.

\section{Gráfico № 2}

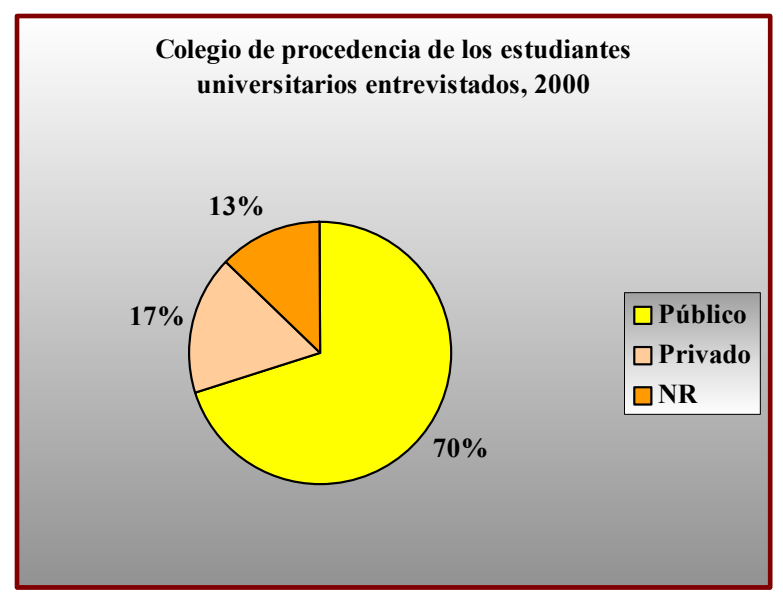

\subsection{Experiencia en lecciones de filosofía.}

De los 390 estudiantes entrevistados el 66\%, es decir 292, recibieron clases de filosofía, y un $30 \%$ asevera no haberlas recibido. Aquellos que no recibieron clases de filosofía corresponden, en la mayoría de los casos, a los que estudiaron en colegios técnicos. Tales datos aseguran que los estudiantes están en posibilidad de valorar el curso de filosofía recibido en quinto año; asimismo, pueden relacionar esa experiencia con la que emerge del curso de filosofía o su similar en la universitaria. Esta información aparece en el gráfico No. 3.

\section{Gráfico № 3}

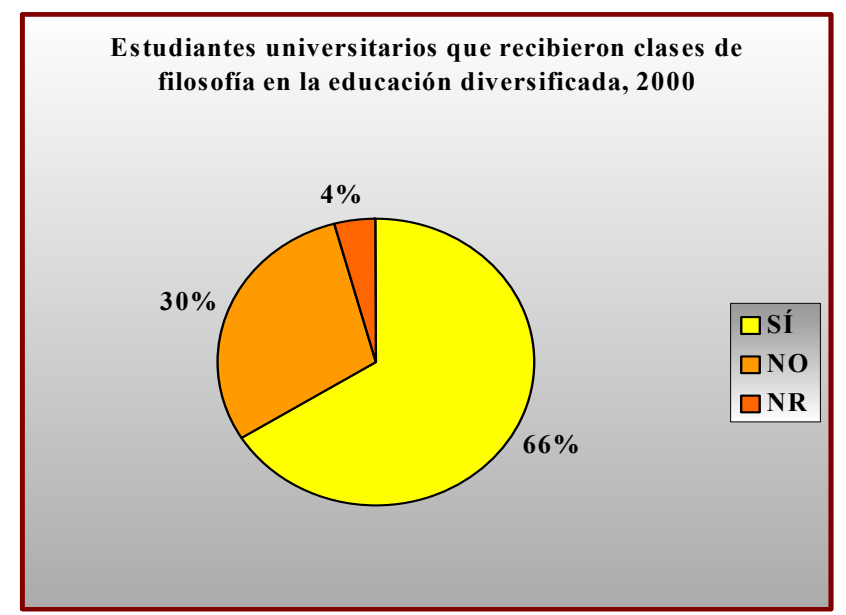


En general, los datos reflejan el predominio de una visión negativa del curso de filosofía en la educación diversificada, cerca la mitad de los estudiantes, esto es el 49\%, este porcentaje representa a 181 estudiantes, quienes aseguran tener una mala experiencia, contra un $30 \%$ (112) que dijo tener una buena experiencia; un $7 \%$ no responde y un $5 \%$ dice no haber recibido lecciones de filosofía, lo cual se ilustra en el gráfico No. 4.

\section{Gráfico No 4}

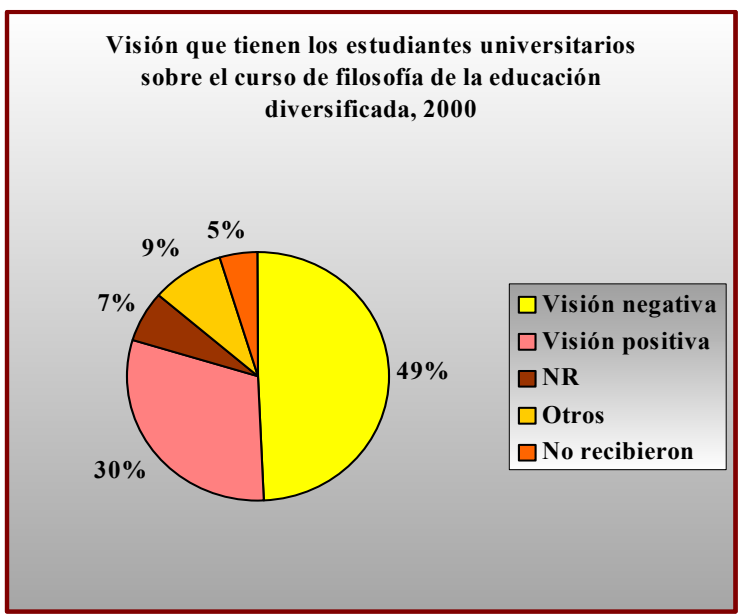

Según las tres grandes categorías, en las que se ordenó la respuesta abierta de los estudiantes la visión negativa, la respuesta se distribuye como sigue: (a) en relación con el curso la visión negativa corresponde a 86 opiniones y la positiva de 56; (b) por el tipo de actividades realizadas, la opinión negativa es de 32 opiniones y la positiva de 22. Además, 17 dijeron no recibir filosofía y 25 no respondieron. (c) Las respuestas en la categoría de percepción del estudiante el número de respuesta negativa es de 23 y la positiva de 14 (d) Un número de 39 repuestas atribuye su opinión negativa al profesor y 19 tienen una visión positiva del profesor.

Las razones o los motivos por los cuales los estudiantes consideran su experiencia como negativa están en relación con tres aspectos valorados: (a) el curso en sí, (b) el profesor y (c) las actividades. Es importante destacar que la visión negativa o positiva del curso tiene responde a la experiencia que marca el docente, especialmente en términos de la formación académica del profesor, la concepción que el profesor tiene de la filosofía y sus metodologías de enseñanza. También, influye la calidad de los materiales didácticos o su inexistencia, los programas vigentes y las condiciones de infraestructura. Algunas opiniones que apoyan sus creencias negativas son: 
(a) sobre el curso:

- $\quad$ De los conocimientos adquiridos lo teórico fue nulo, superficial

- Por ser histórico, aspecto que alude al programa oficial del Ministerio de Educación Pública

- $\quad$ Porque no se vio filosofía sino meditación, relajamiento y técnicas de estudio.

- $\quad$ Poco análisis

- $\quad$ No aprendí nada

- Un curso donde todos aprueban

- $\quad$ No fue un curso amplio sino pinceladas de ciertos cursos relacionados con la posición filosófica

(b) sobre el profesor:

- Aunque conoce de filosofía no sabe impartirla

- $\quad$ El profesor era un psicólogo

- $\quad$ No intentó aumentar la capacidad de razonamiento del estudiante

(c) sobre las actividades

- $\quad$ El estudiante debía apegarse al pensamiento del autor

- $\quad$ Clases aburridas

- $\quad$ Tiempo mal aprovechado

- $\quad$ Se limitó a hablar sobre la vida de los filósofos

- En clase no se explicaban las lecturas

Estas creencias indican que las experiencias en la mediación pedagógica y el programa oficial dificultan un acercamiento crítico a la filosofía, especialmente a su potencial como medio de análisis y reflexión para el proceso de formación estudiantil.

Algunas de las opiniones estudiantiles que constituyen creencias positivas, las cuales coinciden con experiencias más lúdica y analíticas por parte de la mediación docente, son las siguientes:

(a) Sobre el curso:

- $\quad$ Curso muy variado

- $\quad$ Abierto a la discusión y el análisis ${ }^{2}$

- Interesante aprendí diferentes formas de analizar

2 Los estudiantes manifiestan su satisfacción con el curso de filosofía en secundaria cuando se analizan casos. Volumen 4, Número 1, Año 2004 
La opinión negativa se refuerza cuando se relacionan estas experiencias con las de los cursos universitarios. Así, 40 opiniones expresan que el curso de la universidad es mejor que el del colegio; por otra parte, 21 respuestas consideran que el curso de secundaria es peor, y para 7 respuestas no hay diferencia.

Además, la mayoría de las opiniones, es decir, 197 respuestas de los estudiantes tienen una percepción positiva de los cursos de filosofía de formación general o humanística que se imparten en la universidad ${ }^{3}$. Algunos estudiantes consideran que el curso de la universidad es más crítico, profundo, diferente, más teórico y favorece el análisis, se toma en cuenta la opinión de los estudiantes, es difícil, se discuten asuntos cotidianos, tiene relación con la vida. Estas opiniones dependen de la universidad y del curso, por ejemplo, en el ITCR, la opinión positiva es mucho más alta, pues estos versan sobre temas contemporáneos, en la UCR los Seminarios Participativos tiene un gran número favorables, y en la UNA los cursos también versan sobre temas actuales.

Las creencias emergen de experiencias que permiten vincular la formación filosófica con el contexto actual, de ahí su pertinencia, así como por una mayor profundidad que observan en el tiempo destinado a la discusión y análisis filosófico.

La opinión negativa de los estudiantes tiene como causas probables las condiciones de infraestructura, profesores mal preparados, quizás porque no son de la disciplina, los materiales didácticos, la percepción que tienen los jerarcas de los centros educativos sobre la filosofía en su institución, por ende el papel o la importancia que se le asigna al proceso formativo.

En relación con los temas o las áreas de la filosofía estudiados en la educación diversificada, los estudiantes apuntan a tres como las más frecuentes en orden de prioridad: ética, lógica y epistemología, seguido por metafísica, antropología filosófica, filosofía política, ontología, filosofía de la ciencia y filosofía de la tecnología (véase el gráfico $N^{0} 5$ ). Del total de encuestados el 66\%, unos 247 estudiantes, dijeron recordar los temas que estudiaron, el $20 \%$ (75 personas) afirman no recordarlos, y el 14\% no contestaron. Estos datos, muestran una constante en la educación diversificada costarricense, en donde el énfasis en el contenido de los programas de filosofía se centra en la lógica, la ética y la epistemología, aspectos que se encuentra en los planes de estudio del siglo XIX. De lo anterior se desprende además que hay algunos temas a los que los profesores y los planes de estudio

3 El que el curso de la universidad sea mejor que el de secundaria no se desprende que los estudiantes universitarios estén conformes con dichos cursos, pues como se verá dichas visiones varían de una universidad a otro. 
no prestan atención, por ejemplo, la filosofía de la ciencia y la tecnología, según se indica en el gráfico No. 5 .

\section{Gráfico No 5}

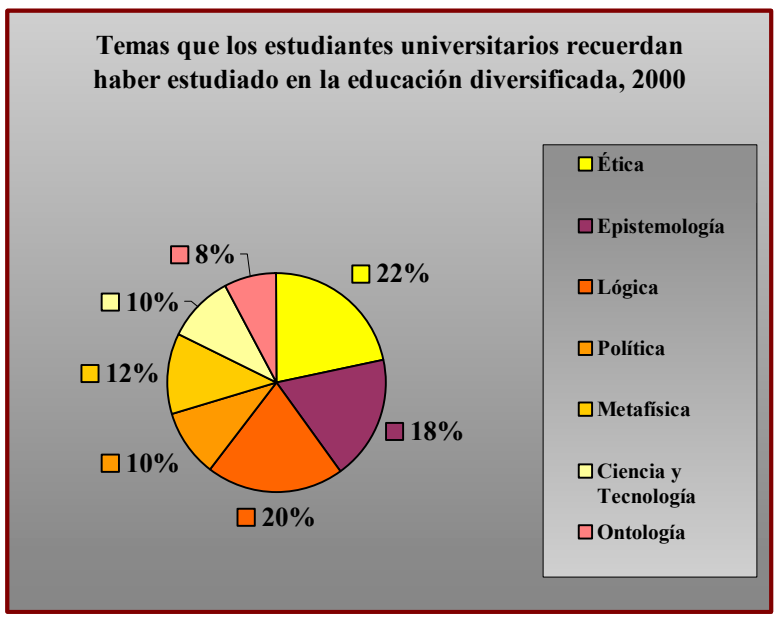

Resulta interesante contrastar los temas analizados con los autores que se estudian en el colegio, lo cual revela que el predominio de los contenidos se centra en la historia de la filosofía clásica. Los autores más citados son: Aristóteles, Sócrates, Platón, curiosamente en filosofía política Karl Marx es uno de los más recordados, seguido por Maquiavelo. Otros autores son: Copérnico, Locke y Kant. Esta información se presenta en el cuadro No.1.

\section{Cuadro $\mathbf{N}^{\circ} 1$}

\section{Autores más citados que los estudiantes universitarios recuerdan haber estudiado en la educación diversificada, 2000}

\begin{tabular}{|l|l|l||c||c|}
\hline \multicolumn{1}{|c|}{ Más de 100: } & Entre $\mathbf{5 0}$ y 100: & $\begin{array}{c}\text { Menos de } \mathbf{5 0} \\
\text { veces: }\end{array}$ \\
\hline Aristóteles & 179 & Maquiavelo & 99 & Habermas \\
\hline Socrátes & 168 & Kant & 88 & Plotino \\
\hline Platón & 156 & Locke & 80 & Mill \\
\hline Marx & 104 & San Agustín & 72 & Hume \\
\hline & Galileo & 70 & Sartre \\
\hline & T. de Aquino & 67 & Popper \\
\hline & Copérnico & 66 & Savater \\
\hline & Hegel & 65 & Russell \\
\hline & Nietzche & 59 & Otros \\
\hline
\end{tabular}


En contraste, los cursos de filosofía de la universidad en relación con la temática estudiada, varían considerablemente a los del colegio. La diversidad depende del centro de estudios y el tipo de curso. Por ejemplo, en la UCR el curso Integrado de Humanidades, Opción Seminario Participativo da énfasis a problemas contemporáneos, mientras que la Opción Regular a la historia del pensamiento; en el ITCR se enfatiza en los problemas éticos de la ciencia y la tecnología, y en la UNA hay una gran diversidad temática. Los resultados que destacan son los siguientes: lógica y argumentación, ética, filosofía y cultura, filosofía de la ciencia y la tecnología, filosofía del desarrollo, ética de la ciencia y la tecnología, filosofía política e historia del pensamiento. No obstante, en la UNA la diversidad temática es tal que da la impresión de poca unidad filosófica en cuanto a los temas abordados. Lo anterior se expresa en el gráfico No. 6.

\section{Gráfico No 6}

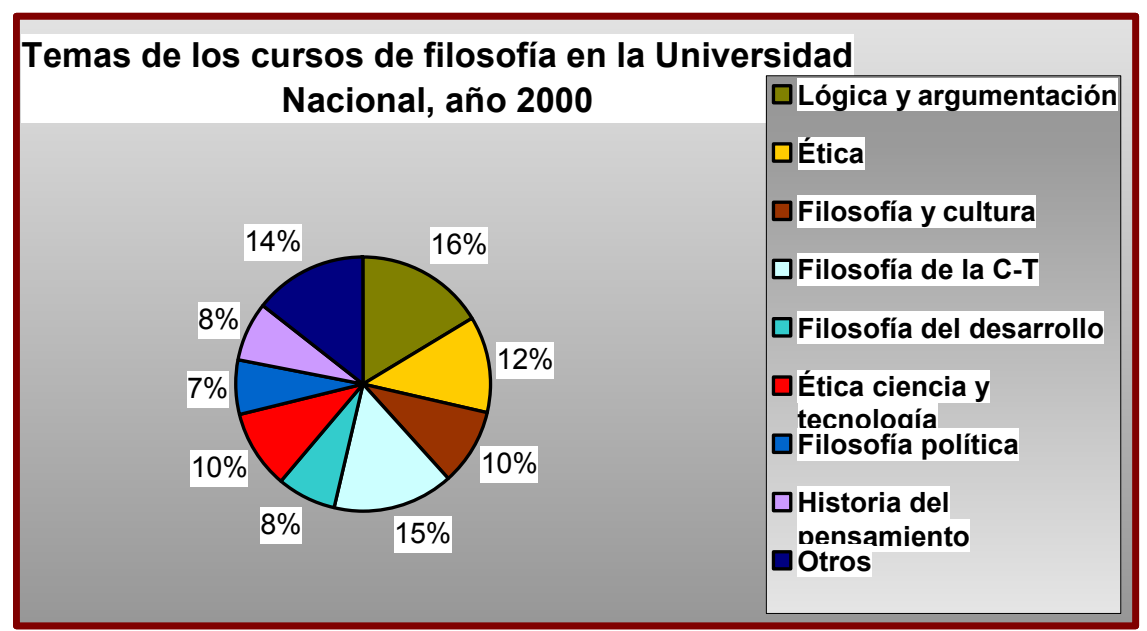

También, hay una gran gama de autores que los estudiantes recuerdan haber estudiado, los más citados son: Rousseau, Platón, Maquiavelo, Aristóteles, Marx y Camacho. Esto parece indicar que en la universidad el énfasis está dado más en los temas que en los autores, a diferencia de la educación diversificada. En las universidades, los estudiantes suelen citar a autores nacionales. En la UCR, a pesar de que hay una diversidad temática y de autores todavía predomina una enseñanza clásica, como se indica en el cuadro No.2. 


\section{Cuadro $N^{\circ} 2$ \\ Autores más citados que los estudiantes de la Universidad de Costa Rica recuerdan haber estudiado en los cursos de filosofía, 2000}

\begin{tabular}{|c|c|c|c|c|}
\hline \multicolumn{2}{|c|}{ Entre 30 y 20 veces: } & \multicolumn{2}{|c|}{ Entre 20 y 10 veces: } & \multirow{2}{*}{$\begin{array}{c}\text { Menos de } 8 \\
\text { veces: } \\
\text { Kant }\end{array}$} \\
\hline Platón & 35 & Aritóteles & 16 & \\
\hline \multirow[t]{7}{*}{ Rousseau } & 30 & Maquiavelo & 19 & Rifkin \\
\hline & & Marx & 14 & Darwin \\
\hline & & Camacho & 12 & Hume \\
\hline & & & & Galileo \\
\hline & & & & Montesquieu \\
\hline & & & & Sócrates \\
\hline & & & & Moro \\
\hline
\end{tabular}

Es importante indicar que las creencias en cuanto temas y autores señaladas por la población estudiantil tanto en el colegio como en la universidad, muestran una continuidad en la visión de la filosofía, en términos de su desarrollo histórico y su relación con áreas afines.

La pregunta sobre cuál es la aplicación de la filosofía, resulta de mucho interés, puesto que intenta conocer el grado de utilidad que el estudiante otorga a dicha disciplina. Los resultados parecen ser halagüeños. Del total de 390 entrevistados, el $72 \%$ considera la filosofía tiene una aplicabilidad en su vida; sólo el $21 \%$, es decir 70 estudiantes, no respondieron la pregunta (39 de la UNA, 38 de la UCR y 2 del ITCR), y únicamente el 7\%, esto es 22 personas, dijeron que la filosofía no tiene ninguna aplicación. Por lo cual, se infiere que la mayoría de las estudiantes perciben un beneficio en recibir clases de filosofía, como aparece en el gráfico No. 7. 


\section{Gráfico $N^{\circ} 7$}

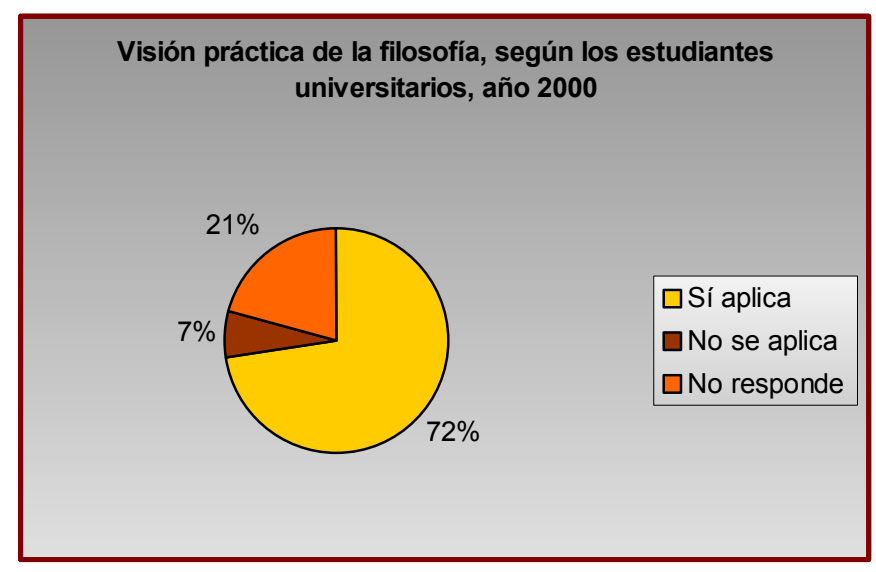

La finalidad práctica se desglosa como sigue: en la vida cotidiana 128 respuestas (44\%), fundamentalmente, se refieren a los aspectos éticos aplicados al mejoramiento de las relaciones interpersonales y a sus vidas cotidianas; le sigue el ámbito profesional y académico con 74 respuestas (26\%); luego, según estas opiniones la filosofía les aporta una visión de mundo, para un total de 39 respuestas (14\%), a la filosofía misma con 34 respuestas $(4 \%)$ y a todo 10 respuestas (véase el gráfico $N^{\circ} 8$ ).

\section{Gráfico No 8}

Aplicación de la filosofía según ámbito, año 2000

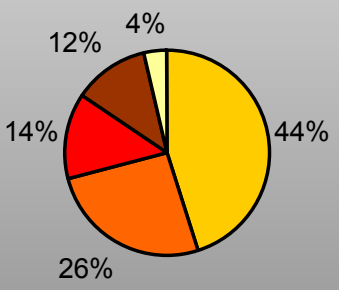

\section{口Vida cotidiana \\ 口Profesional y académico}

$\square$ Visión de mundo

口En sí misma

$\square$ Todas las anteriores

Es importante notar que esta vinculación práctica de la filosofía emerge de una percepción relacionada con los aportes que puede brindar para la construcción de juicios morales, la incorporación de conocimientos para el desempeño profesional y la capacidad de análisis y crítica que promueve la reflexión filosófica, en relación con este aspecto se alude al cambio en la visión de mundo. 
Con base en los cursos de filosofía recibidos se les pidió a los estudiantes que intentaran definir la filosofía a partir de sus experiencias, las respuestas muestran una diversidad de maneras de concebirla. Del total de 390 entrevistas, un 27\%, es decir, 105 estudiantes no responden a esta pregunta, lo cual puede ser un indicador de que no tienen una imagen precisa acerca del significado de la filosofía. Un 52\%, es decir, 203 estudiantes, logran formarse una noción de lo qué es la filosofía, y un $21 \%$ tan sólo indican atributos positivos o negativos de la filosofía (18 en la UNA, 14 en el ITCR y 50 en la UCR). Lo anterior refuerza la idea de la imagen difusa de lo que es la filosofía; este es un aspecto importante para retomar en el ámbito curricular, porque, al menos, en la educación diversificada es fundamental que la población estudiantil tenga un acercamiento a la filosofía, que le permita explicar y comprender qué es la filosofía como campo de producción del conocimiento y el proceso de construcción del pensamiento filosófico. Este aspecto es importante porque la primera unidad del programa de estudios de educación diversificada parece abocarse a este aspecto, en la práctica no ofrece los resultados esperados. En el caso de la Filosofía es conveniente indicar la relevancia que tiene el personal docente para conducir el proceso pedagógico y orientar la visión de la filosofía que coincida con las preferencias académicas que defiende el personal docente. Lo anterior se agrava cuando este personal no cuenta con la formación específica en el campo mencionado.

Las respuestas de los estudiantes se organizaron en dos categorías: (A) la primera, responde a la pregunta ¿qué es...?, la respuesta: una ciencia, un arte, un método, entre otros; (B) la segunda, responde a la pregunta ¿qué hace...?, es decir, se enfoca a su función, esta perspectiva se fija en las palabras claves más citadas. Esto aporta dos énfasis que contribuyen al análisis del contenido. Según (A) la tendencia dominante es considerar la filosofía como:

1. Una ciencia o una rama de la ciencia, con 84 respuestas $(52 \%)$. Esta percepción es predominante en la UCR (54 opiniones) y en la UNA (21 opiniones). Este es un enfoque tradicional de concebir la filosofía.

2. Un estudio, análisis, reflexión sobre la realidad con 35 respuestas (21\%). Esta opinión se encuentra entre estudiantes de la UCR y del ITCR.

3. Una disciplina, corriente que busca explicar u ofrecer una visión de mundo, con 19 respuestas (12\%). La conciben de esta manera los estudiantes del ITCR.

4. Una visión de mundo, con 11 respuestas (7\%). Esta percepción es de los estudiantes del ITCR. 
5. Un medio o un método para alcanzar algo, con 7 respuestas (4\%). Sólo los estudiantes del ITCR.

6. Un arte, con 7 respuestas (4\%). Sólo los estudiantes de la UCR. Apréciese los porcentajes relativos en el gráfico $\mathrm{N}^{\circ} 9$.

\section{Gráfico $N^{\circ} 9$}

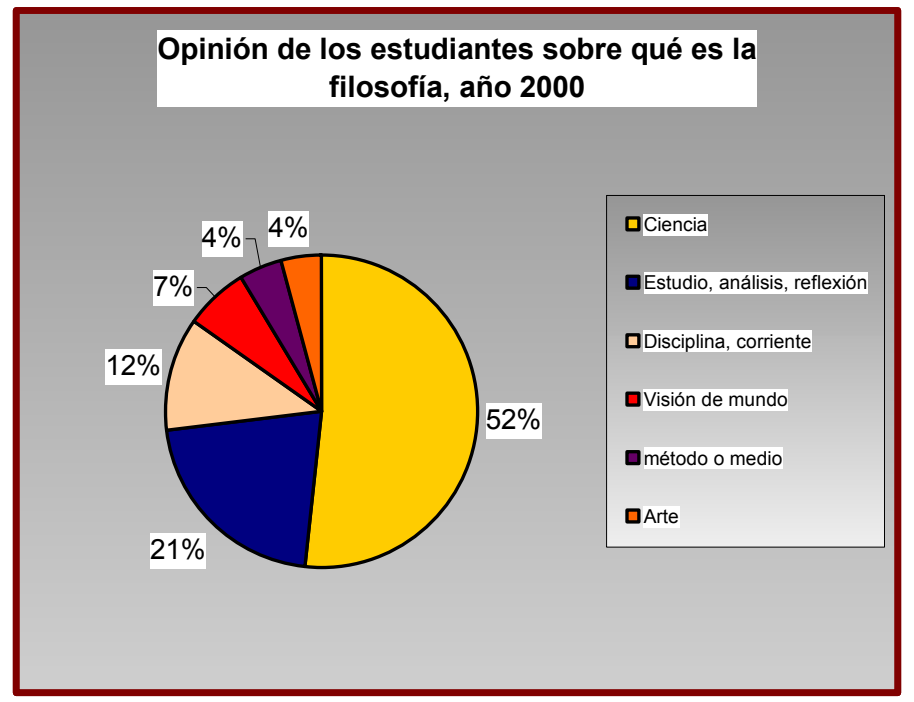

Según la función de la filosofía percibida por la población estudiantil, se pone énfasis en que la filosofía contribuye a pensar, reflexionar, analizar y explicar el mundo.

- Se hace referencia a un medio o manera o método para llegar a tener criterio propio, que ayuda a hacer análisis profundo. A veces se le concibe como un instrumento que enseña a pensar.

- La filosofía se concibe como un estudio o un análisis acerca del mundo, ya sea social, científico, tecnológico, entre otros.

- Otro aspecto en que se agrupan la mayor cantidad de palabras claves es "explicar". El "explicar" tiene que ver con la idea de que la filosofía interroga el por qué de las cosas, sean físicas, sociales o éticas. La filosofía intenta dar una respuesta a esas interrogantes, en particular esta opinión se encuentra en los estudiantes del ITCR.

- La filosofía es una manera o aporta maneras diferentes de ver o plantearse las cosas, situaciones, problemas, entre otros. Muchos también mencionan que en ellos produjo un cambio de visión o de punto de vista, particularmente esto es señalado por los estudiantes del ITCR. 
Si bien en la mayoría de los casos la filosofía se la define como una ciencia, su función es la de ayudar a pensar, explicar, estudiar, reflexionar, analizar, dar respuesta a interrogantes, aporta otra visión acerca del mundo, la vida social, la cotidianidad, la ciencia y la tecnología, con lo cual se entiende la filosofía no como una disciplina científica propiamente dicha.

\section{Conclusión}

Existe en la percepción de los estudiantes universitarios una visión negativa de la enseñanza de la filosofía en la educación diversificada costarricense. Esta imagen mejora en los cursos universitarios, en contraste con la enseñanza recibida en el colegio. Sin embargo, esta apreciación positiva no es uniforme, depende de la universidad, el tipo de curso y del personal docente. En relación con lo anterior, los cursos que aparecen mejor valorados por la población estudiantil, son los del ITCR y los Seminarios Participativos de la UCR.

Los estudiantes prefieren cursos con temas de actualidad en donde predomine el análisis crítico y reflexivo, en los que se promueva la participación del estudiante. También, exigen que los métodos de enseñanza filosófica sean dinámicos y flexibles.

A pesar de estas dificultades los estudiantes consideran que la filosofía es importante para su desarrollo académico, profesional y su vida personal, pues les brinda nuevas visiones o formas de abordar el mundo en que se desenvuelven.

Las creencias y las percepciones de la población estudiantil en relación con las experiencias de filosofía en las que han sido partícipes en el colegio y en la universidad, reflejan la importancia de revisar, analizar, reflexionar y proponer una propuesta curricular para la asignatura de Filosofía que comprenda un proceso pedagógico más acorde con el sujeto de la educación diversificada, es decir, quién es y qué espera de su permanencia en el sistema educativo formal; asimismo cómo se percibe la filosofía por parte del personal docente, las autoridades educativas, los especialistas y la sociedad costarricense. En este sentido, consideramos que una experiencia positiva y académica en la formación filosófica en la educación diversificada es clave para repensar y fortalecer dicha experiencia en la educación superior. 


\section{REFERENCIAS}

Accorinti, S. (1999) Introducción a Filosofía para Niños. Buenos Aires: Ediciones Manantial.

Carvajal, A.; García, J.; Alfaro, M.; Triana, M.; Rodríguez, C. (2001). La situación de la enseñanza de la filosofía en la educación diversificada costarricense. San José, C. R.: Vicerrectoria de Investigación/Universidad de Costa Rica.

Cifuentes, Luis; Gutiérrez, José (coord.). (1997). Enseñar y aprender filosofía en la educación secundaria. Barcelona: ICR/Universität/Horsori.

Dewey, J. (1998). Democracia y Educación. Madrid: Ediciones Morata.

Elliott, John. (1994). Investigación-acción en educación. $2^{\text {a }}$ ed. Madrid: Morata.

Foment, Eudaldo. (1991). Enseñanza de la filosofía en la educación secundaria. Madrid: Rialp.

Gómez, Mario. (1988). Didáctica de la filosofía. $2^{\mathrm{a}}$ ed., Santa Fe de Bogotá: Universidad Santo Tomás.

Guiyart, Rosa Ma (ed.). (1999). La enseñanza de la filosofía hoy. VI (20), Barcelona: GRAO/ Francese-Torrego.

Longás, A. (et al.). (1997). Aspectos didácticos de la filosofía. Zaragoza: ICE/Universidad de Zaragoza.

Ordoñez, Jacinto. (1983). A propósito de la enseñanza de la filosofía. En: Praxis, 27-28: 99-110, julio-diciembre.

Rodrigo, María, Rodriguez, A, Marrero, Javier. (1993). Las teorías implícitas. Una aproximación al conocimiento cotidiano. Madrid: Visor Distribuciones.

Santiuste, Víctor. (1984). Didáctica de la filosofía: teoría, métodos, programas y evaluación. Madrid: Marcea.

Splitter, L. \& Sharp, A. (1996) La otra educación: Filosofía para niños y la comunidad de indagación. Buenos Aires: Ediciones Manantial.

Tiana, Alejandro. (1996). "La evaluación de los sistemas educativos". En Revista Iberoamericana, $\mathrm{N}^{0} 10$ : 37-61.

Valverde, Francisco (1999). Programa de Filosofía. San José, C.R.: Ministerio de Educación Pública. 


\section{Anexo $\mathrm{N}^{\circ} 1$ \\ UNIVERSIDAD DE COSTA RICA INSTITUTO DE INVESTIGACIONES FILOSÓFICAS \\ PROYECTO:}

"Situación de la enseñanza de la filosofía en la educación diversificada costarricense"

Encuesta de opinión a los estudiantes universitarios sobre la enseñanza de la filosofía en los colegios

Estimado (a) estudiante:

La presente encuesta tiene como propósito solicitarle su amable colaboración para obtener información valiosa con el propósito de realizar un diagnóstico de la enseñanza de la filosofía en los colegios del país, por lo que le rogamos contestar las siguientes preguntas:

$\begin{array}{lll}\text { 1. Sexo: 1. ( ) Masculino } & \text { 2.( ) Femenino }\end{array}$

2. Edad: 1. ( ) Menos de 20 años

2. ( ) De 20 a 25 años

4. ( ) Más de 25 años

5. ( ) NS/NR

3. Colegio de Procedencia:

4. Curso de filosofía en el que está matriculado:

5. Indique la carrera en que está empadronado (matriculado):

6. Nivel de la Carrera (aquel en que tiene más materias):
1. ( ) Primer año
4. ( ) Cuarto año
2. ( ) Segundo año
5. ( ) Quinto año
3. ( ) Tercer años
6. ( ) NS/NR

7. Trabaja:

1. ( ) No

2. ( ) Sí

Jornada:

1. ( ) Por horas

2. ( ) Tiempo parcial

3. ( ) Tiempo completo

4. ( ) NS/NR

8. Recibió clases de filosofía en su colegio: 1. ( ) Sí 2 2. ( ) No

(pase a la pregunta 13) 
9. Describa brevemente cómo fue su experiencia en el curso de filosofía que recibió en el colegio.

10. ¿Recuerda usted los temas y autores que estudiaron en ese curso?
1. ( ) Sí
2. ( ) No

Si su respuesta es afirmativa, responda cuáles temas y autores reconoce en la siguiente lista. Marque con una equis $(x)$ en el espacio en blanco

\section{Temas}

1. ( ) Ética

2. ( ) Filosofía política

3. ( ) Epistemología

4. ( ) Lógica

5. ( ) Filosofía de la ciencia

6. ( ) Filosofía de la tecnología

7. ( ) Metafísica

8. ( ) Ontología

9. ( ) Antropología filosófica

10. ( ) Otro:

\section{Autores}

1. ( ) Habermas 12.( ) Socrátes

2. ( ) Maquiavelo 13.( ) Nietzche

3. ( ) Plotino 14.( ) Savater

4. ( ) Copérnico 16.( ) Hegel

5. ( ) Mill

6. ( ) Aristóteles 18.( ) Marx

7. ( ) Locke 19.( ) Platón

8. ( ) Hume 20.( ) Galileo

9. ( ) Sartre 21.( ) Kant

10.( ) Pooper 22.( ) Leibniz

11.( ) San Agustín 23.( ) Sto. Tomás

24.( ) Otros:

11. A partir de su experiencia en el curso de filosofía del colegio, señale cuáles diferencias encuentra con el curso de filosofía que se le ofrece en la universidad.

Anote los temas o autores que haya estudiado en el curso de filosofía de la universidad:

Temas

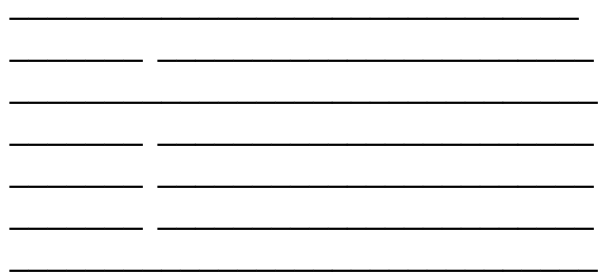

Autores

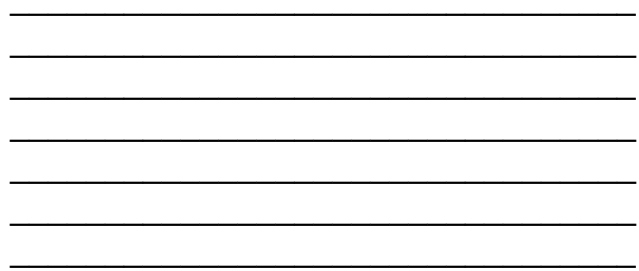


13. ¿Con qué aspectos de su vida cotidiana, académica, profesional; entre otros, puede usted relacionar los conocimientos obtenidos sobre filosofía? Explique o aporte ejemplos.

14. Con base en los cursos recibidos cómo conceptualizaría la filosofía: 